\title{
ADAPTIVE BEAM TRACKING FOR INTERFERENCE ALIGNMENT IN TIME-VARYING MIMO INTERFERENCE CHANNELS: CONJUGATE GRADIENT APPROACH
}

\author{
Junse Lee, Heejung Yu, Youngchul Sung ${ }^{\dagger}$ and Yong H. Lee
}

\begin{abstract}
Based on a linear formulation to interference alignment, an adaptive algorithm for interference-aligning beam tracking in time-varying MIMO interference channels is proposed. It is shown that obtaining the interferencealigning beam vector is equivalent to minimizing a certain Rayleigh quotient, and the conjugate gradient approach is adopted to construct an adaptive algorithm. The convergence and stability of the proposed algorithm are established in static channel case, and numerical results show that the proposed algorithm performs well compared with other existing methods with much less complexity.
\end{abstract}

Index Terms- Interference alignment, adaptive algorithm, conjugate gradient, least squares

\section{INTRODUCTION}

Interference alignment (IA) achieves the maximum degree of freedom (DoF) in multiuser interference channels [1], and is an attractive solution to handle interference properly in future wireless networks. Since its introduction much research has been performed on the achievability issue from information-theoretical perspective, e.g., $[1,2]$. In addition to this effort, there has also been a line of research on the invention of efficient beam design algorithms [3-6] from signal processing perspective and such invention is important to realize the potential of IA in practice. In particular, $\mathrm{Yu}$ et al. proposed an efficient beam tracking algorithm for IA in time-varying multi-input multi-output (MIMO) interference channels [6] based on their previous work [5] of least squares approach to IA. Their method of beam tracking is based on the matrix perturbation approach. That is, the beam solution at the next time is an additive update of the current time and the difference is derived from the channel perturbation based on the eigenvector perturbation theory. Even if their proposed method is computationally effective, it is not a purely adaptive algorithm, and requires a full eigendecomposition periodically to provide a reference beam vector to which perturbation term is added at each time step while tracking. In this paper, we propose a purely adaptive algorithm for the beam design for IA which works in both static and slowlyvarying MIMO interference channels for the first time to the best of our knowledge. Our approach to an adaptive algorithm is also based on our previous work [5] of least squares approach to IA, but we here use the congugate gradient descent [7] to update the beam vector rather than try to obtain the next beam vector by computing the perturbed eigenvector. The convergence of the proposed

$\dagger$ Junse Lee, Youngchul Sung, and Yong H. Lee are with Dept. of Electrical Engineering, KAIST, South Korea. Email:\{junselee, ysung@ee., yohlee@ee.\}kaist.ac.kr. Heejung Yu is with ETRI, South Korea. Email:heejung@etri.re.kr. This research was supported by Basic Science Research Program through the National Research Foundation of Korea (NRF) funded by the Ministry of Education, Science and Technology (2010-0021269) and IT R \& D program of MKE/KEIT [KI001835, "5G mobile communication systems based on beam division multiple access and relays with group cooperation"] adaptive algorithm is established in the static channel, and its performance is evaluated numerically and compared with other existing methods. It is shown that the proposed algorithm works well up to reasonable mobile speed and yields almost the same performance as the previous algorithms with much less computational complexity. (Throughput this paper, we use the same notation as in [5].)

\section{SYSTEM MODEL AND BACKGROUND}

We consider a $K$-user $M \times N$ MIMO interference channel in which $K$ transmitter-receiver pairs exist and transmitters and receivers have $N$ and $M$ antennas $(N \geq M)$, respectively. Assuming fully connected interference structure, the received signal at time $n$ at receiver $k$ is given by

$$
\mathbf{y}_{k}[n]=\mathbf{H}_{k k}[n] \mathbf{V}_{k}[n] \mathbf{s}_{k}[n]+\sum_{l=1, l \neq k}^{K} \mathbf{H}_{k l}[n] \mathbf{V}_{l}[n] \mathbf{s}_{l}[n]+\mathbf{n}_{k}[n],
$$

where $\mathbf{H}_{k l}[n]$ is the $M \times N$ MIMO channel matrix at time $n$ from transmitter $l$ to receiver $k, \mathbf{V}_{l}[n]$ and $\mathbf{s}_{l}[n]$ are the $N \times d_{l}$ beamforming matrix and $d_{l} \times 1$ signal vector at transmitter $l$, respectively. $\mathbf{n}_{k}[n] \sim \mathcal{C N}\left(0, \sigma^{2} \mathbf{I}\right)$ is a zero-mean complex Gaussian noise vector. We assume $d=d_{1}=\cdots=d_{K}$, and further assume that the channel information is known to the transmitters and receivers.

2.1. Background: LS approach to interference alignment [5] In this subsection, we briefly introduce the previous work relevant to our new beam design in the next section. It was shown in [5] that the necessary and sufficient IA condition could be expressed by a system of linear equations with dummy variables, given by

$$
\tilde{\mathbf{H}}[n] \mathbf{v}[n]=\mathbf{0},
$$

where $\tilde{\mathbf{H}}$ is defined as (3), and

$$
\mathbf{v}[n] \triangleq\left[\operatorname{vec}\left(\mathbf{V}_{1}[n]\right)^{T}, \operatorname{vec}\left(\mathbf{V}_{2}[n]\right)^{T}, \cdots, \operatorname{vec}\left(\mathbf{V}_{K}[n]\right)^{T}\right]^{T} .
$$

The key advantage of this formulation is that the dummy variables $\left\{\mathbf{A}_{k l}[n]\right\}$ inside $\tilde{\mathbf{H}}[n]$ are given in closed-form by

$\mathbf{A}_{1 l}[n]=\left\{\left(\mathbf{H}_{1 l}[n] \mathbf{V}_{l}[n]\right)^{\dagger} \mathbf{H}_{12}[n] \mathbf{V}_{2}[n]\right\}^{T}, l=3, \cdots, K$,
$\mathbf{A}_{k l}[n]=\left\{\left(\mathbf{H}_{k l}[n] \mathbf{V}_{l}[n]\right)^{\dagger} \mathbf{H}_{k 1}[n] \mathbf{V}_{1}[n]\right\}^{T}, k, l=2, \cdots, K, k \neq l$,

when $\mathbf{v}[n]$ is given. The existence of solution $\mathbf{v}[n]$ and $\left\{\mathbf{A}_{k l}[n]\right\}$ to (2) depends on the size $\frac{K(K-2) M^{2}}{2} \times \frac{K M N}{2}$ of $\tilde{\mathbf{H}}[n]$. There are three distinct cases under the linear formulation with dummy variables. First, when $\tilde{\mathbf{H}}[n]$ is strictly fat, as in an example of $K=3$, $M=2, N=3$ and $d=1$, there exists a non-trivial solution regardless of the choice of $\left\{\mathbf{A}_{k l}[n]\right\}$ given by a null vector of $\tilde{\mathbf{H}}[n]$. When $\tilde{\mathbf{H}}[n]$ is tall (including square), on the other hand, the case is further divided into two subcases depending on the rank of $\tilde{\mathbf{H}}[n]$. 


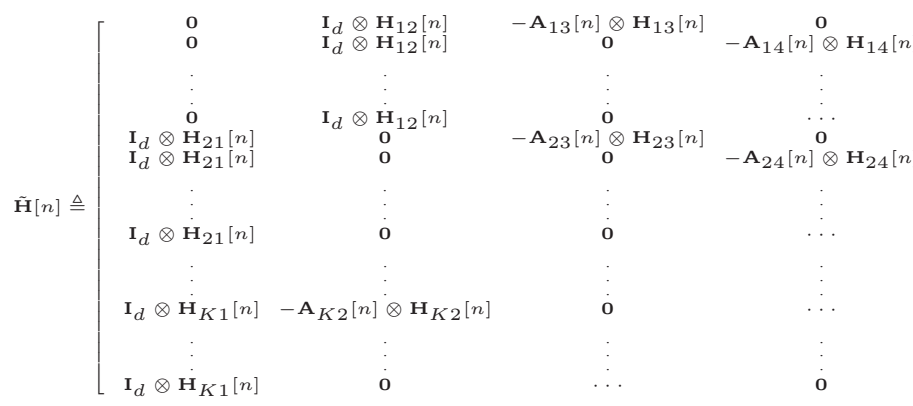

The first subcase is that $\tilde{\mathbf{H}}[n]$ is rank-deficient with properly chosen $\left\{\mathbf{A}_{k l}[n]\right\}$ as in an example of $K=3$ and $M=N=2 d$. In the second subcase, $\tilde{\mathbf{H}}[n]$ has full-column rank for any choice of $\left\{\mathbf{A}_{k l}[n]\right\}$ which corresponds to the case that the perfect IA is infeasible. In such a case, an approximate solution can be found by minimizing the interference misalignment $\|\tilde{\mathbf{H}}[n] \mathbf{v}[n]\|$. Although the algorithm based on this linear formulation (2) of IA was proposed, the equivalence of this linear formulation with dummy variable to the bilinear formulation in [3] and the existence of solution $d \geq 2$ were not established in [5]. Here, we provide a new result on this aspect in the feasible case of $K=3$ and $M=N=2 d$. (It was proved in [1] that the perfect IA was not feasible for $K \geq 4$ in $M \times M$ MIMO channels.)

Theorem 1 In the feasible case of $K=3$ and $M=N=2 d$, the matrix $\tilde{\mathbf{H}}[n]$ is rank-deficient by nullity $d$ with a proper set of $\left\{\mathbf{A}_{k l}[n]\right\}$ almost surely for randomly realized $\left\{\mathbf{H}_{k l}[n]\right\}$ from a continuous distribution.

Proof: Please see appendix.

Theorem 1 provides a basis that the interference-aligning beam vector can be found by solving (2) and (5) for any $d=M / 2$. The solution is easily obtained by solving

$$
\hat{\mathbf{v}}[n]=\underset{\|\mathbf{v}[n]\|=1}{\arg \min }\|\tilde{\mathbf{H}}[n] \mathbf{v}[n]\|^{2}=\underset{\|\mathbf{v}[n]\|=1}{\arg \min } \mathbf{v}^{H}[n] \mathbf{R}[n] \mathbf{v}[n]
$$

and (5) iteratively with an initialization, where $\mathbf{R}[n] \triangleq \tilde{\mathbf{H}}^{H}[n] \tilde{\mathbf{H}}[n]$ It was proved and shown in [5] that this iterative algorithm converges and this algorithm converges much faster than the previous algorithm based on the bilinear formulation. In the feasible case, indeed $\tilde{\mathbf{H}}[n]^{(i)}$ at the $i$-th iteration becomes rank-deficient by order $d$ as the iteration number increases. It is seen in case of $d \geq 2$ that the subvectors (of one null vector $\mathbf{v}[n]$ ) corresponding to multiple streams of one user are identical. However, there are $d$ linearly independent null vectors and thus $d$ linearly independent beam vectors per user are achieved by the proposed method. Note that the solution to (6) is given by the eigenvector of $\mathbf{R}[n]$ corresponding to the smallest eigenvalue, and this fact provided a basis for an efficient beam tracking algorithm based on the eigenvector perturbation theory in [6]. That is, as channel varies over time, $\mathbf{R}[n+p]=\tilde{\mathbf{H}}^{H}[n+p] \tilde{\mathbf{H}}[n+p]=\mathbf{R}[n]+\mathbf{G}_{n}[n+p]$ $(p \geq 1)$ and the extreme eigenvector of $\mathbf{R}[n+p]$ can be obtained as a perturbed extreme eigenvector of $\mathbf{R}[n]$ when the perturbation term $\mathbf{G}_{n}[n+p]$ is small. Here, one full eigendecomposition and correct dummy variables are required for $\mathbf{R}[n]$ and then for $p=1,2, \cdots, D$ only additive update based on $\mathbf{G}_{n}[n+p]$ is performed. (This process is repeated with period $D+1$ over time.) This tracking method reduces computational complexity significantly and yields a good performance when the tracking depth $D$ is chosen well [6].

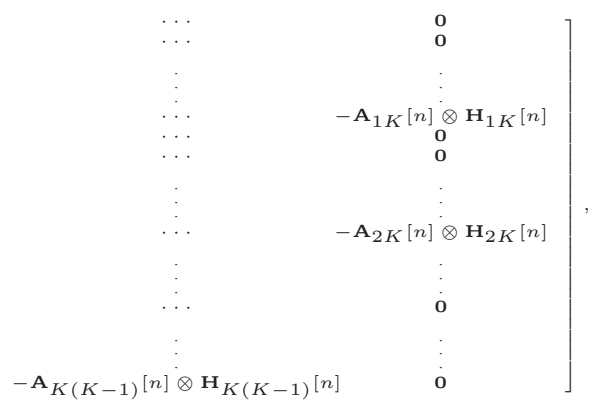

(3)

\section{ADAPTIVE BEAM TRACKING FOR IA}

In this section, we present a new purely adaptive algorithm for interference-aligning beam vector design considering $d=1$. Note that finding the minimum eigenvalue and corresponding eigenvector of the Hermitian matrix $\mathbf{R}[n]$ in (6) is equivalent to obtaining the minimum value of the Rayleigh quotient

$$
R(\mathbf{R}[n], \mathbf{v}[n]) \triangleq<\mathbf{v}[n], \mathbf{R}[n] \mathbf{v}[n]>/\|\mathbf{v}[n]\|^{2},
$$

where $\langle\cdot, \cdot\rangle$ denotes the inner product between two vectors. Thus, our beam solution can also be obtained using descent methods applied to minimize the Rayleigh quotient (7). Among various descent methods, we adopt the conjugate gradient (CG) approach which shows fast convergence and does not require matrix inversion as the Netwon descent [7]. In combination with the dummy variable update (5), the CG provides a very effective adaptive algorithm for interference alignment in static and time-varying channels, described below.

Algorithm 1: The Conjugate Gradient algorithm for Interference Alignment (CGIA)

Initialization

- $\operatorname{Set}\left(\mathbf{A}_{k l}=\mathbf{I}\right)$ and set initial $\mathbf{v}[0]$. for $n=1,2, \cdots$

- Construct $\tilde{\mathbf{H}}[n]$ with $\left\{\mathbf{A}_{k l}\right\}$ and $\left\{\mathbf{H}_{k l}[n]\right\}$

- Update $\mathbf{v}[n]$ by running the CG subroutine below: $\mathbf{v}[n]=$ CG subroutine $(\mathbf{v}[n-1], \tilde{\mathbf{H}}[n])$

- Obtain $\left\{\mathbf{V}_{l}[n]\right\}$ by reshaping and properly normalizing $\mathbf{v}[n]$.

- if $\bmod \left(n, N_{1}\right)=0$ \}

$$
\text { - Update }\left\{\mathbf{A}_{k l}\right\} \text { using (5). }
$$

$\mathrm{v}^{\prime}=$ CG subroutine $(\mathrm{v}, \tilde{\mathrm{H}}) \quad$ [7]

$\{$ Initialization

- Set $\mathbf{x}(0)=\mathbf{v}, b(0)=0$ and $\mathbf{R}=\tilde{\mathbf{H}}^{H} \tilde{\mathbf{H}}$

- $\lambda(0)=<\mathbf{x}(0), \mathbf{R x}(0)>/\|\mathbf{x}(0)\|^{2}$

for $k=0,1, \cdots, N_{2}-1$

- if $\mathrm{k}=0$

$$
\diamond \mathbf{r}(0)=\mathbf{p}(0)=[\lambda(0) \mathbf{x}(0)-\mathbf{R} \mathbf{x}(0)] /\|\mathbf{x}(0)\|^{2}
$$

- $t(k)=\left[-B+\sqrt{B^{2}-4 C D}\right] /(2 D)$, where

$\diamond P_{a}(k)=<\mathbf{p}(k), \mathbf{R x}(k)>/\|\mathbf{x}(k)\|^{2}$

$\diamond P_{b}(k)=<\mathbf{p}(k), \mathbf{R} \mathbf{p}(k)>/\|\mathbf{x}(k)\|^{2}$

$\diamond P_{c}(k)=<\mathbf{p}(k), \mathbf{x}(k)>/\|\mathbf{x}(k)\|^{2}$

$\diamond P_{d}(k)=<\mathbf{p}(k), \mathbf{p}(k)>/\|\mathbf{x}(k)\|^{2}$

$\diamond D=P_{b}(k) P_{c}(k)-P_{a}(k) P_{d}(k)$

$\diamond B=P_{b}(k)-\lambda(k) P_{d}(k)$ 


$$
\begin{aligned}
\diamond C & =P_{a}(k)-\lambda(k) P_{c}(k) \\
\bullet & \mathbf{x}(k+1)=\mathbf{x}(k)+t(k) \mathbf{p}(k) . \\
\bullet \lambda(k+1) & =<\mathbf{x}(k+1), \mathbf{R x}(k+1)>/\|\mathbf{x}(k+1)\|^{2} \\
\bullet & \mathbf{r}(k+1)=[\lambda(k+1) \mathbf{x}(k+1)-\mathbf{R x}(k+1)] /\|\mathbf{x}(k+1)\|^{2} \\
\bullet b(k+1) & =-<\mathbf{r}(k+1), \mathbf{R} \mathbf{p}(k)>/<\mathbf{p}(k), \mathbf{R} \mathbf{p}(k)> \\
& \bullet \mathbf{p}(k+1)=\mathbf{r}(k+1)+b(k+1) \mathbf{p}(k) \\
\mathbf{v}^{\prime}=\mathbf{x}\left(N_{2}\right) . &
\end{aligned}
$$

In the above CG subroutine, $\mathbf{r}$ is the residual, $\mathbf{p}$ is the direction vector for update and $\mathbf{x}$ is the desired vector. Depending on the normalization variation, several different computations of $b(k)$ are available for $\mathrm{CG}$, but simulations showed almost the same performance for the variations. In Algorithm 1, we further have freedom to design $\left(N_{1}, N_{2}\right)$, where $N_{1}$ is the period of the dummy variable update and $N_{2}$ is the number of CG updates per time step. Since the CG finds the extreme eigenvector with $\operatorname{size}(\mathbf{R}[n])$ updates, $\left(N_{1}=1, N_{2}=\operatorname{size}(\mathbf{R}[n])\right)$ and $\left(N_{1}=\operatorname{size}(\mathbf{R}[n]), N_{2}=1\right)$ result in the same solution which is the extreme eigenvector of $\mathbf{R}[n]$ when the channel is static, and the CGIA with these combinations of $\left(N_{1}, N_{2}\right)$ is equivalent to the iterative least squares algorithm proposed in [5]. ${ }^{1}$ For time-varying channels, $\left(N_{1}, N_{2}\right)$ can be optimized to yield the best performance.

\section{ANALYSIS}

In this section, we provide several properties of the proposed CGIA algorithm. First we establish the convergence of CGIA.

Theorem 2 The CGIA converges for any initialization and $\left(N_{1}, N_{2}\right)$ for static channels, i.e., $\mathbf{H}_{k l}[0]=\cdots=\mathbf{H}_{k l}[n]$ for all $n$. Thus, the algorithm is stable for static channels.

Proof: The proof is by showing that the Rayleigh quotient $R$ decreases monotonically. Given $\mathbf{A}_{k l}$, it is known that the CG update does not increase the Rayleigh quotient [8]. Also, given $\mathbf{v}$, it was proved in [5] that the dummy variable update (5) does not increase the Rayleigh quotient since (5) itself is the least squares solution to minimize the Rayleigh quotient as a function of $\mathbf{A}_{k l}$, i.e.,

$$
R\left(\tilde{\mathbf{H}}\left(\mathbf{H}_{k l}, \mathbf{A}_{k l}\right), \mathbf{v}^{\prime}\right) \geq R\left(\tilde{\mathbf{H}}\left(\mathbf{H}_{k l}, \mathbf{A}_{k l}^{\prime}\right), \mathbf{v}^{\prime}\right),
$$

where $\mathbf{A}_{k l}^{\prime}$ is the updated $\mathbf{A}_{k l}$ based on the new beam $\mathbf{v}^{\prime}$ from the CG step. Combining the two facts, the Rayleigh quotient is monotone decreasing for CGIA regardless of the value of $\left(N_{1}, N_{2}\right)$, but the Rayleigh quotient is lower bounded by zero. Thus, CGIA converges by the monotone convergence theorem.

The CGIA algorithm updates the beam vector so that the Rayleigh quotient (7) or equivalently the overall interference misalignment $\|\tilde{\mathbf{H}}[n] \mathbf{v}[n]\|$ under the norm constraint is minimized. As the Rayleigh quotient (7) decreases, we expect that the interference leakage also decreases. This can be proved in the feasible case of $K=3$ and $2 d=M=N=2$. The interference leakage $\gamma_{k}$ at receiver $k$ is defined in [3] as $\gamma_{k} \triangleq \frac{\sum_{i=d+1}^{2 d} \lambda_{i}\left(\boldsymbol{\Gamma}_{k}\right)}{\sum_{i=1}^{2 d} \lambda_{i}\left(\boldsymbol{\Gamma}_{k}\right)}$, where $\lambda_{i}\left(\boldsymbol{\Gamma}_{k}\right)$ are the eigenvalues of the interference covariance matrix at receiver $k, \boldsymbol{\Gamma}_{k} \triangleq \sum_{l \neq k} \mathbf{H}_{k l} \mathbf{V}_{l} \mathbf{V}_{l}^{H} \mathbf{H}_{k l}^{H}$, and $\lambda_{i+1}\left(\boldsymbol{\Gamma}_{k}\right) \leq \lambda_{i}\left(\boldsymbol{\Gamma}_{k}\right)$ for all $i$. Thus, in the case of $2 d=M=N=2$ we have $\gamma_{k}=\frac{\lambda_{2}\left(\boldsymbol{\Gamma}_{k}\right)}{\lambda_{1}\left(\boldsymbol{\Gamma}_{k}\right)+\lambda_{2}\left(\boldsymbol{\Gamma}_{k}\right)}$. On the other hand, the Rayleigh quotient is given by the minimum eigenvalue $R$ satisfying

$$
\tilde{\mathbf{H}} \mathbf{v}=R \mathbf{v} .
$$

\footnotetext{
${ }^{1}$ In [7], the numerical results show that the algorithm does not find the exact solution but almost same solution in $\operatorname{size}(\mathbf{R}[n])$ steps.
}

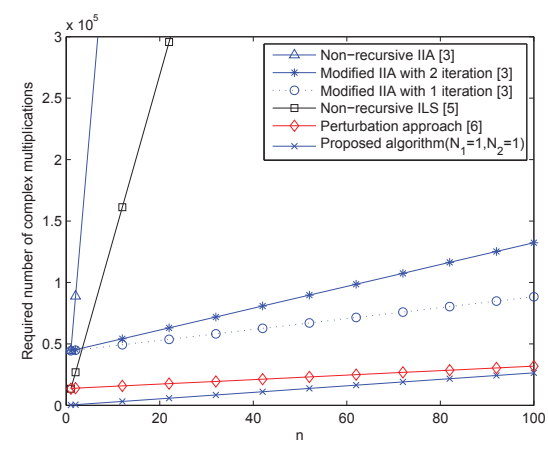

Fig. 1. Complexity $(K=3,2 d=M=N=2)$

Regarding the relationship between $\gamma_{k}$ and $R$ we have the following result.

Theorem 3 Suppose that the convergent beam solution by CGIA has a norm strictly larger than zero for each user. Then in case of $K=3$ and $2 d=M=N=2$ there exists a constant $c$ such that

$$
\gamma_{k} \leq c|R|^{2}
$$

where $R$ is the Rayleigh quotient and $\gamma_{k}$ is the corresponding interference leakage.

The proof is by direct computation of the eigenvalues $\lambda_{1}\left(\boldsymbol{\Gamma}_{k}\right)$, $\lambda_{2}\left(\boldsymbol{\Gamma}_{k}\right)$ and $\gamma_{k}$ and representing $\gamma_{k}$ in terms of $R$ exploiting the structure of $\tilde{\mathbf{H}}$ in (3) and (8).

Now we compare the computational complexity of beam design algorithms for interference alignment in time-varying channels. We use the number of complex multiplication as the criterion. Fig. 1 shows the complexity of various algorithms. We considered the iterative method in [3] and its two modifications to time-varying scenario, the iterative least squares in [5] and the perturbation approach in [6]. For CGIA we used $N_{1}=N_{2}=1$. For all other methods than CGIA, at $n=1$ the complexity shows the complexity for static channel. The number of iterations at $n=1$ for two non-recursive methods [3] and [5] was set to 100 and 50, respectively, so that further iterations do not enhance the performance for each. For the non-recursive curves, the same iteration is repeated at each time step with new channel value and thus the complexity increases with the largest slopes as time goes. However, the clever algorithms such as the perturbation algorithm [6] and the modifications of [3] drastically reduces the complexity. (In the modifications of [3] the previous beam vector was used as the initial value for iterations and one or two iterations were only applied. It was observed that at least two iterations were required to yield similar performance. (The curve with one iteration was shown to provide the computational performance limit of the method.) Although the perturbation method in [6] has smaller slope than CGIA, it requires a jump at every $m D, m=1,2, \cdots$. Thus, the relative complexity between the two algorithms depends on the value of $D$. (The initial steps for CGIA to converge is negligible as time goes.)

\section{NUMERICAL RESULTS}

Here, we provide some numerical results of the performance of CGIA. We considered $K=3$ and $2 d=M=N=2$ MIMO interference channel. We generated a 1st-order Gauss-Markov channel process for each channel tap with the carrier frequency of $1 \mathrm{GHz}$ 


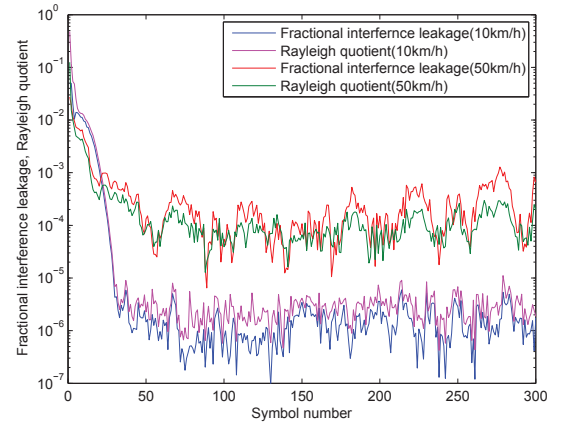

Fig. 2. Tracking performance $(K=3,2 d=M=N=2)$

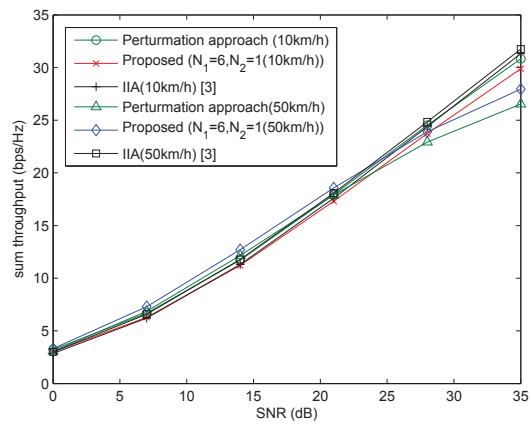

Fig. 3. Sum rate performance $(K=3,2 d=M=N=2)$

and symbol duration of $66.7 \mu \mathrm{sec}$ corresponding to the symbol duration of 3GPP long-term evolution (LTE). Fig. 2 shows the tracking performance of the CGIA for the time-varying channel. As expected the algorithm converges initially and there exists steadystate floor of the Rayleigh quotient and interference leakage due to the time-variations of the channel with larger floor for higher mobile speed. It is also seen that the Rayleigh quotient and interference leakage behaves in the same manner as predicted by Theorem 3. Finally, Fig. 3 shows the sum rate performance of CGIA with $N_{1}=6$ and $N_{2}=1$, the perturbation method [6] with $D=100$ and the original iterative method $^{2}$ in [3] as the performance reference. As expected, there is tracking loss at high SNR as the mobile speed increases. (See the curves corresponding to $50 \mathrm{~km} / \mathrm{h}$.) It is seen that the CGIA performs better than the perturbation method [6] at $50 \mathrm{~km} / \mathrm{h}$ whereas the perturbation method performs better at the lower speed of $10 \mathrm{~km} / \mathrm{h}$. This is because $D=100$ is small enough for $10 \mathrm{~km} / \mathrm{h}$ but it is large at $50 \mathrm{~km} / \mathrm{h}$. Thus, from the complexity in Fig. 1 we see that the perturbation method has advantage at low mobile speed whereas the CGIA has at high mobile speed.

\section{CONCLUSION AND FUTURE WORK}

In this paper, we have proposed the CGIA algorithm for purely adaptive beam tracking for interference alignment in time-varying MIMO interference channels. We have established the conver-

\footnotetext{
${ }^{2}$ At each time step, 100 iterations were performed to yield almost perfect interference-aligning beam vectors for the channel at each time step.
}

gence of the CGIA at static channels and have evaluated its performance in time-varying channel case numerically. The CGIA shows comparable performance with significant complexity reduction and it can be used for practical systems with real time-varying channels to realize interference alignment when channel state information is available at transmitters. Future work includes the extension to the case of $d \geq 2$ and further analysis of steady-state leakage as a function of mobile speed.

\section{REFERENCES}

[1] V. R. Cadambe and S. A. Jafar, "Interference alignment and degrees of freedom of the $K$-user interference channel," IEEE Trans. Inform. Theory, vol. 54, pp. 3425 - 3441, Aug. 2008.

[2] A. Ghasemi, A. S. Motahari, and A. K. Khandani, "Interference alignment for the $K$ user MIMO interference channel," in Proc. IEEE ISIT, Austin, TX, June 2010.

[3] K. Gomadam, V. R. Cadambe, and S. A. Jafar, "Approaching the capacity of wireless networks through distributed interfernce alignment," ArXiv pre-print cs.IT/0803.3816, Mar. 2008.

[4] S. W. Peters and R. W. Heath, "Interference alignment via alternating minimization," in Proc. IEEE ICASSP, Taipei, Taiwan, Apr. 2009.

[5] H. Yu and Y. Sung, "Least squares approach to joint beam design for interference alignment in multiuser multi-input multi-output interference channels," IEEE Trans. Signal Process., vol. 58, pp. 4960 - 4966, Sep. 2010.

[6] H. Yu, Y. Sung, H. Kim, and Y. H. Lee, "Adaptive beam tracking for interference alignment for multiuser time-varying MIMO interference channels," in Proc. IEEE ICASSP, Dallas, TX, Mar. 2010.

[7] X. Yang, T. Sarkar, and E. Arvas, "A survey of conjugate gradient algorithms for solution of extreme eigen-problems of a symmetric matrix," IEEE Transactions on Acoustics Speech and Signal Processing, vol. 37, no. 10, pp. 1550-1556, 1989.

[8] I. Shavitt, C. Bender, A. Pipano, and R. Hosteny, "The iterative calculation of several of the lowest or highest eigenvalues and corresponding eigenvectors of very large symmetric matrices," Journal of Computational Physics, vol. 11, p. 90, 1973.

Appendix: Proof of Theorem 1 It suffices to show that there exist a full columnrank matrix $\mathbf{V}$ (with size of $K M \times d)$ and $\left\{\mathbf{A}_{k l}\right\}$ such that $\tilde{\mathbf{H}} \mathbf{V}=0$. $(\operatorname{rank}(\mathbf{V})$ cannot be greater than $d$ by the known feasibility result [1].) By partitioning $\mathbf{V}$ into $\left[\mathbf{V}_{1}^{T}, \mathbf{V}_{2}^{T}, \mathbf{V}_{3}^{T}\right]^{T}$, the IA condition given by $\tilde{\mathbf{H}} \mathbf{V}=0$ can be rewritten as

$$
\begin{aligned}
& \mathbf{I}_{d} \otimes \mathbf{H}_{12} \mathbf{V}_{2}-\mathbf{A}_{13} \otimes \mathbf{H}_{13} \mathbf{V}_{3}=\mathbf{0}, \\
& \mathbf{I}_{d} \otimes \mathbf{H}_{21} \mathbf{V}_{1}-\mathbf{A}_{23} \otimes \mathbf{H}_{23} \mathbf{V}_{3}=\mathbf{0}, \\
& \mathbf{I}_{d} \otimes \mathbf{H}_{31} \mathbf{V}_{1}-\mathbf{A}_{32} \otimes \mathbf{H}_{32} \mathbf{V}_{2}=\mathbf{0} .
\end{aligned}
$$

For randomly realized channel $\left\{\mathbf{H}_{k l}\right\}$, the matrices are invertible almost surely. With (11) and (12), (10) is rewritten by $\left(\mathbf{A}_{23} \mathbf{A}_{13}^{-1} \mathbf{A}_{32}^{-1}\right) \otimes\left(\mathbf{H}_{21}^{-1} \mathbf{H}_{23} \mathbf{H}_{13}^{-1} \mathbf{H}_{12} \mathbf{H}_{32}^{-1}\right.$ $\left.\mathbf{H}_{31}\right) \mathbf{V}_{1}=\mathbf{V}_{1}$ since $(\mathbf{A} \otimes \mathbf{B})^{-1}=\mathbf{A}^{-1} \otimes \mathbf{B}^{-1}$ for invertible matrices $\mathbf{A}$ and $\mathbf{B}$, and $(\mathbf{A} \otimes \mathbf{B})(\mathbf{C} \otimes \mathbf{D})=\mathbf{A} \mathbf{C} \otimes \mathbf{B D}$. Defining $\mathbf{A}^{\prime} \triangleq \mathbf{A}_{23} \mathbf{A}_{13}^{-1} \mathbf{A}_{32}^{-1}$ and $\mathbf{F} \triangleq \mathbf{H}_{21}^{-1} \mathbf{H}_{23} \mathbf{H}_{13}^{-1} \mathbf{H}_{12} \mathbf{H}_{32}^{-1} \mathbf{H}_{31}$, we obtain

$$
\left(\mathbf{A}^{\prime} \otimes \mathbf{F}\right) \mathbf{V}_{1}=\mathbf{V}_{1} \mathbf{I}
$$

Note that (13) is an invariant subspace equation. Assume that the sets of eigenvalues and eigenvectors of $\mathbf{A}^{\prime}$ and $\mathbf{F}$ are $\left\{\lambda_{A^{\prime}, 1}, \cdots \lambda_{A^{\prime}, d}\right\},\left\{\mathbf{a}_{1}, \cdots, \mathbf{a}_{d}\right\}$, and $\left\{\lambda_{F, 1}, \cdots, \lambda_{F, M}\right\},\left\{\mathbf{f}_{1}, \cdots, \mathbf{f}_{M}\right\}$, respectively. (Since the set of complex $M \times M$ matrices that are not diagonalizable has measure zero in the space of $\mathbb{C}^{M \times M}$, the randomly generated matrix $\mathbf{F}$ is diagonalizable almost surely and its $M$ eigenvectors are linearly independent.) Suppose now that $\mathbf{A}^{\prime}$ have the $d$ eigenvalues in the set of inverse eigenvalues of $\mathbf{F}$, i.e., $\lambda_{A^{\prime}, m}=\frac{1}{\lambda_{F, \pi(m)}}$, where $\{\pi(1), \cdots, \pi(d)\} \subset\{1, \cdots, M\}$ for some permutation $\pi(\cdot)$. Then, $\mathbf{A}^{\prime} \otimes \mathbf{F}$ have a unit eigenvalue with geometric multiplicity $d$ and the corresponding eigenvectors are $\mathbf{a}_{1} \otimes \mathbf{f}_{\pi(1)}, \cdots, \mathbf{a}_{d} \otimes \mathbf{f}_{\pi(d)}$ which are also linearly independent by the linear independence of $\mathbf{f}_{\pi(m)}$ and the property of the Kronecker product since $\mathbf{a}_{m}, m=1, \cdots, d$ have at least one non-zero element. The matrix composed of these eigenvectors satisfies (13). The existence of such an $\mathbf{A}^{\prime}$ is straightforward using singular value decomposition (SVD) and there also exist $\mathbf{A}_{k l}$ such that $\mathbf{A}^{\prime}=\mathbf{A}_{23} \mathbf{A}_{13}^{-1} \mathbf{A}_{32}^{-1}$, e.g., $\mathbf{A}_{13}=\mathbf{A}_{32}=\mathbf{I}$ and $\mathbf{A}_{23}=\mathbf{A}^{\prime}$. Since a submatrix $\mathbf{V}_{1}$ of $\mathbf{V}$ has rank $d, \mathbf{V}$ has also rank $d$. 